\title{
HYPEREXPANSIVE OPERATOR VALUED UNILATERAL WEIGHTED SHIFTS
}

\author{
ZENON JAN JABŁOŃSKI \\ Instytut Matematyki, Uniwersytet Jagielloński, ul. Reymonta 4, PL-30059 Kraków \\ e-mail: jablonsk@im.uj.edu.pl
}

(Received 3 September, 2003; accepted 18 November, 2003)

\begin{abstract}
The notion of a completely hyperexpansive operator has been introduced in [1] by Athavale. In this paper hyperexpansive operator valued unilateral weighted shifts are investigated. A unique semispectral measure is associated with a bounded completely hyperexpansive operator valued unilateral weighted shift with invertible weights. Examples of bounded and unbounded hyperexpansive operator valued unilateral weighted shifts with invariant domains are given.
\end{abstract}

2000 Mathematics Subject Classification. Primary 47A30, 47A20; Secondary 47A57, 47B20.

Introduction. It is well known that subnormal operators are closely related to the theory of positive definite functions on the abelian semigroup $\left(\mathbb{N},+, n^{*}=n\right)$, and completely hyperexpansive operators to negative definite functions on $\left(\mathbb{N},+, n^{*}=n\right)$ (cf. [1]). More precisely, a bounded operator $T$ is a subnormal contraction (resp. a completely hyperexpansive operator) if and only if $\left\{\left\|T^{n} f\right\|^{2}\right\}_{n=0}^{\infty}$ is a completely monotone (resp. completely alternating) sequence for all $f \in \mathcal{H}$.

Notice also that the class of 2-hyperexpansive operators has "small" intersections with some other known classes of operators. Its intersection with the class of paranormal operators is equal to the class of isometries. Also, the intersection of the classes of 2-hyperexpansive operators and the Toeplitz operators on the Hardy space is the class of isometrics, see $[\mathbf{4 , 5}]$. Recall that if $T$ is a $k$-isometric (resp. completely or $k$-hyperexpansive) operator, then so are all its power $T^{n}$, for $n \geqslant 1$ (cf. [5, Theorem 2.3]).

The aim of the present paper is to show that there exist bounded and unbounded densely defined hyperexpansive operator valued unilateral weighted shifts with invariant domains that form an essentially new class of hyperexpansive operators (cf. Section 5).

In Section 2 we consider bounded and unbounded 2-isometric and hyperexpansive operator valued unilateral weighted shifts and characterize the hyperexpansive ones. In Section 3 we give a useful description of 2-isometric operator valued unilateral weighted shift with invertible weights. In Section 4 we describe the $\mathrm{CH}$-minimal spectral dilation of a bounded completely hyperexpansive operator valued unilateral weighted shift with invertible weights. In this section we also consider the problem of hyperexpansive completions. Finally, Section 5 contains various examples of hyperexpansive operator valued unilateral weighted shifts. 
1. Hyperexpansive operators. All operators taken into consideration in this paper are assumed to be linear. Let $\mathcal{H}$ be a (complex) Hilbert space. Given a family $\left\{X_{J}\right\}_{J \in J}$ of subsets of $\mathcal{H}$ we denote by $\bigvee_{j \in J} X_{J}$ the closed linear span of $\bigcup_{J \in J} X_{J}$. From now on $\boldsymbol{B}(\mathcal{H})$ stands for the $\mathrm{C}^{*}$-algebra of all bounded operators on $\mathcal{H} ; I_{\mathcal{H}}$ stands for the identity operator on $\mathcal{H}$. By an operator in $\mathcal{H}$ we understand a linear mapping $T: \mathcal{H} \supset \mathcal{D}(T) \rightarrow \mathcal{H}$ defined on a linear subspace $\mathcal{D}(T)$ of $\mathcal{H}$ which is called the domain of $T$. Denote by $\mathcal{R}(T)$ the range of the operator $T$.

For an operator $T$ in $\mathcal{H}$ we set

$$
\Theta_{T, n}(f):=\sum_{0 \leq p \leq n}(-1)^{p}\left(\begin{array}{l}
n \\
p
\end{array}\right)\left\|T^{p} f\right\|^{2} \quad\left(f \in \mathcal{D}\left(T^{n}\right), n \geqslant 1\right) .
$$

Let us recall that an operator $T$ in $\mathcal{H}$ is (cf. [1], [5], [9], [12])

(a) k-isometry $(k \geqslant 1)$ if $\Theta_{T, k}(f)=0$ for $f \in \mathcal{D}\left(T^{k}\right)$,

(b) $k$-expansive $(k \geqslant 1)$ if $\Theta_{T, k}(f) \leq 0$ for $f \in \mathcal{D}\left(T^{k}\right)$,

(c) $k$-hyperexpansive $(k \geqslant 1)$ if $\Theta_{T, n}(f) \leq 0$ for $f \in \mathcal{D}\left(T^{n}\right)$ and $n=1, \ldots, k$,

(d) completely hyperexpansive if $\Theta_{T, n}(f) \leq 0$ for $f \in \mathcal{D}\left(T^{n}\right)$ and $n \geqslant 1$.

According to Richter's result (cf. [9, Lemma 1]) the notions of 2-expansivity and 2hyperexpansivity coincide in the case of bounded operators. Moreover, in the bounded case the inclusion relations among the classes defined above are as follows:

2 - isometry $\subset$ completely hyperexpansive $\subset \ldots \subset(k+1)$ - hyperexpansive $\subset k$-hyperexpansive $\subset \ldots \subset 1-$ hyperexpansive,

and all these inclusions are proper (cf. [9] and [12]).

Denote by $\mathcal{B}([0,1])$ the $\sigma$-algebra of all Borel subsets of $[0,1]$. By a semispectral measure on $[0,1]$ we understand a positive operator valued Borel measure $G$ : $\mathcal{B}([0,1]) \rightarrow \boldsymbol{B}(\mathcal{H})$ that is $\sigma$-additive in the weak operator topology (we do not assume that $G([0,1])=I$ ). By the Naimark dilation theorem (cf. [8, Theorems 4.1 and 6.4]) every semispectral measure has a minimal spectral dilation. Moreover, if $\left(\mathcal{K}_{1}, R_{1}, E_{1}\right)$ and $\left(\mathcal{K}_{2}, R_{2}, E_{2}\right)$ are minimal spectral dilations of $G$, then there is a (unique) unitary isomorphism $U: \mathcal{K}_{1} \rightarrow \mathcal{K}_{2}$ such that

$$
\begin{aligned}
U E_{1}(\sigma) & =E_{2}(\sigma) U, \quad \sigma \in \mathcal{B}([0,1]), \\
U R_{1} & =R_{2} .
\end{aligned}
$$

We say that $G$ is commutative if $G(\sigma) G(\tau)=G(\tau) G(\sigma),(\sigma, \tau \in \mathcal{B}([0,1]))$. If moreover $G([0,1])=I$ and $G(\sigma)$ is an orthogonal projection for every $\sigma \in \mathcal{B}([0,1])$, then $G$ is called a spectral measure. We say that a triplet $(\mathcal{K}, R, E)$ is a minimal spectral dilation of a semispectral measure $G: \mathcal{B}([0,1]) \rightarrow \boldsymbol{B}(\mathcal{H})$, if $\mathcal{K}$ is a Hilbert space, $R \in \boldsymbol{B}(\mathcal{H}, \mathcal{K})$, where $\boldsymbol{B}(\mathcal{H}, \mathcal{K})$ is the algebra of bounded linear operators from $\mathcal{H}$ into $\mathcal{K}$, and $E: \mathcal{B}([0,1]) \rightarrow \boldsymbol{B}(\mathcal{K})$ is a spectral measure such that

$$
\begin{gathered}
G(\sigma)=R^{*} E(\sigma) R, \quad \sigma \in \mathcal{B}([0,1]), \\
\mathcal{K}=\bigvee_{\sigma \in \mathcal{B}([0,1])} E(\sigma) R(\mathcal{H}) .
\end{gathered}
$$


Our first observation follows from the Stone-Weierstrass approximation theorem and (automatic) regularity of a semispectral measure on the interval $[0,1]$.

Lemma 1.1. Let $\mathcal{K}$ be a Hilbert space, $R \in \boldsymbol{B}(\mathcal{H}, \mathcal{K})$ and $E: \mathcal{B}([0,1]) \rightarrow \boldsymbol{B}(\mathcal{K}) a$ spectral measure. Then $(\mathcal{K}, R, E)$ is a minimal spectral dilation of a semispectral measure $G: \mathcal{B}([0,1]) \rightarrow \boldsymbol{B}(\mathcal{H})$ if and only if

$$
\begin{aligned}
\int_{[0,1]} x^{n} G(\mathrm{~d} x) & =R^{*} \int_{[0,1]} x^{n} E(\mathrm{~d} x) R \quad(n \geqslant 0), \\
\mathcal{K} & =\bigvee_{n \geqslant 0} \int_{[0,1]} x^{n} E(\mathrm{~d} x) R(\mathcal{H}) .
\end{aligned}
$$

It is shown in [5] that an operator $T \in \boldsymbol{B}(\mathcal{H})$ is completely hyperexpansive if and only if there exists a unique semispectral measure $G: \mathcal{B}([0,1]) \rightarrow \boldsymbol{B}(\mathcal{H})$ such that

$$
T^{* n} T^{n}=I+\int_{[0,1]}\left(1+\cdots+x^{n-1}\right) G(\mathrm{~d} x), \quad n \geqslant 1 .
$$

Recall that a triplet $(\mathcal{K}, R, A)$ is a $C H$-minimal spectral decomposition of $T$ if $\mathcal{K}$ is a Hilbert space, $R \in \boldsymbol{B}(\mathcal{H}, \mathcal{K}), A \in \boldsymbol{B}(\mathcal{K})$ is an operator such that $0 \leq A \leq I$ and

$$
\begin{aligned}
T^{* n} T^{n}= & I+R^{*} \sum_{j=0}^{n-1} A^{j} R \quad(n \geqslant 1), \\
& \bigvee_{n \geqslant 0} A^{n} R(\mathcal{H})=\mathcal{K} .
\end{aligned}
$$

It is also shown in [5] that an operator $T \in \boldsymbol{B}(\mathcal{H})$ is completely hyperexpansive if and only if it has a $\mathrm{CH}$-minimal spectral decomposition (which is unique up to unitary equivalence).

Let $\phi$ be a real-valued function on the additive semigroup $\mathbb{N}$ of all non-negative integers. We let the difference operator $\nabla$ act on $\phi$ through the formula

$$
(\nabla \phi)(s)=\phi(s)-\phi(s+1) .
$$

The relations $\nabla^{0} \phi=\phi$ and $\nabla^{n} \phi=\nabla \nabla^{n-1} \phi$ inductively define $\nabla^{n}$ for all $n \geqslant 0$. A realvalued function $\psi$ on $\mathbb{N}$ is said to be completely alternating if $\left(\nabla^{n} \psi\right)(s) \leq 0$ for all $s \geqslant 0$ and $n \geqslant 1$. A sequence of operators $\left\{A_{n}\right\}_{n=0}^{\infty} \subseteq \boldsymbol{B}(\mathcal{H})$ is said to be completely alternating if for all $f \in \mathcal{H}$ the scalar sequence $\left\{\left\langle A_{n} f, f\right\rangle\right\}_{n=0}^{\infty}$ is completely alternating.

The following result may be proved in much the same way as [5, Theorem 4.2].

THEOREM 1.2. A sequence of operators $\left\{A_{n}\right\}_{n=0}^{\infty} \subseteq \boldsymbol{B}(\mathcal{H})$ is completely alternating if and only if there exists a semispectral measure $F$ on $[0,1]$ such that

$$
A_{n}=A_{0}+\int_{[0,1]}\left(1+\cdots+x^{n-1}\right) F(\mathrm{~d} x) \quad(n \geqslant 1) .
$$

Moreover, a semispectral measure $F$ is uniquely determined by (1.2).

2. Hyperexpansive operator valued unilateral weighted shifts. In this section we give a characterization of hyperexpansive unilateral weighted shifts. 
Let $l^{2}(\mathcal{H})=\oplus_{n=0}^{\infty} \mathcal{H}$ be the orthogonal sum of $\aleph_{0}$ copies of the Hilbert space $\mathcal{H}$ with a scalar product defined by

$$
\langle f, g\rangle=\sum_{i=0}^{\infty}\left\langle f_{n}, g_{n}\right\rangle, \quad f=\oplus_{n=0}^{\infty} f_{n} \in l^{2}(\mathcal{H}), g=\oplus_{n=0}^{\infty} g_{n} \in l^{2}(\mathcal{H}),
$$

and let $\left\{T_{i}\right\}_{i=0}^{\infty}$ be a sequence of bounded linear operators on $\mathcal{H}$. The operator $T$ in $l^{2}(\mathcal{H})$ defined by

$$
\begin{gathered}
\mathcal{D}(T)=\left\{f \in l^{2}(\mathcal{H}): \sum_{n=0}^{\infty}\left\|T_{i} f_{i}\right\|^{2}<\infty\right\}, \\
T\left(f_{0}, f_{1}, f_{2}, \ldots\right)=\left(0, T_{0} f_{0}, T_{1} f_{1}, T_{2} f_{2}, \ldots\right), \quad f \in \mathcal{D}(T),
\end{gathered}
$$

is called an operator valued unilateral weighted shift with weights $\left\{T_{i}\right\}_{i=0}^{\infty}$. The operator valued unilateral weighted shift $T$ is closed and $T$ is bounded if and only if the sequence $\left\{\left\|T_{n}\right\|\right\}_{n=0}^{\infty}$ is bounded; if this happens, then $\|T\|=\sup _{n \geqslant 0}\left\|T_{n}\right\|$. If all operators $T_{n}$, $n \geqslant 0$ are invertible and the sequence $\left\{\left\|T_{n}\right\|\right\}_{n=0}^{\infty}$ is bounded, then we shall say that $T$ is an invertibly weighted shift (cf. [7]). The set of all invertibly weighted shifts on $l^{2}(\mathcal{H})$ is denoted by $l_{I W}^{2}(\mathcal{H})$.

The following lemma is stated without proof, as it differs only in notation from the proofs in $[6,7]$ of the corresponding results for scalar matrices and invertibly weighted shifts.

LeMma 2.1. Let $T, S \in \boldsymbol{B}\left(l^{2}(\mathcal{H})\right)$ be operator valued weighted shifts with weights $\left\{T_{n}\right\}_{n=0}^{\infty}$ and $\left\{S_{n}\right\}_{n=0}^{\infty}$, respectively, and suppose that the operators $T_{n}$ and $S_{n}$ have dense ranges for all $n \geqslant 0$. Then the following are equivalent.

(i) $U S=T U$, for some unitary operator $U$.

(ii) $U$ is diagonal with entries $\left\{U_{n}\right\}_{n=0}^{\infty}$ and $U_{i+1} S_{i}=T_{i} U_{i}$, for all $i \geqslant 0$.

It is well known (cf. [7, Theorem 3.4]) that if $T$ is an invertibly weighted shift, then $T$ is unitarily equivalent to a weighted shift with positive weights. The following result enables us to characterize 2 -isometric and completely hyperexpansive invertibly weighted shifts.

Proposition 2.2. Let $T \in l_{I W}^{2}(\mathcal{H})$ be an operator valued weighted shift with weights $\left\{T_{n}\right\}_{n=0}^{\infty}$. Then $T$ is unitarily equivalent to an operator valued weighted shift $T^{\prime} \in l_{I W}^{2}(\mathcal{H})$ with weights $\left\{T_{n}^{\prime}\right\}_{n=0}^{\infty}$, where $T_{n}^{\prime} \ldots T_{0}^{\prime}$ are positive, for all $n \geqslant 0$.

The set of all invertibly weighted shifts $S$ on $l^{2}(\mathcal{H})$ with the property that all products $S_{n} \ldots S_{0}, n \geqslant 0$, are positive, is denoted by $l_{I W P}^{2}(\mathcal{H})$.

Proof. Let $T_{0}=U_{0} P_{0}$ be the polar decomposition of $T_{0}$. Then $S_{0} \stackrel{\text { df }}{=} U_{0}^{*} T_{0}=P_{0}$ is positive and invertible and $U_{0}$ is unitary. Now we proceed by induction. If the invertible operators $S_{0}, \ldots, S_{n-1}$ and the unitary operators $U_{0}, \ldots, U_{n-1}$ are defined, let

$$
V_{n} P_{n}=T_{n} U_{n-1} S_{n-1} \ldots U_{0} S_{0}
$$

be the polar decomposition of the operator $T_{n} U_{n-1} S_{n-1} \ldots U_{0} S_{0}$, and let

$$
U_{n} \stackrel{\mathrm{df}}{=} V_{n}\left(U_{n-1} \ldots U_{0}\right)^{*} .
$$


Then $P_{n}$ is positive and invertible, $U_{n}$ is unitary and $S_{n} \stackrel{\text { df }}{=} U_{n}^{*} T_{n}$ is invertible. Let us define $S \stackrel{\text { df }}{=} \oplus_{n=0}^{\infty} S_{n}$ and let $U \stackrel{\text { df }}{=} \bigoplus_{n=0}^{\infty} U_{n}$. If $U_{+}$denotes the unilateral shift given by $U_{+}\left(h_{0}, h_{1}, \ldots\right)=\left(0, h_{0}, h_{1}, \ldots\right)$, then $U_{+} \in l_{I W}^{2}(\mathcal{H})$ and $T=U_{+} U S$. Let us define the unitary operator $W$ with diagonal weights $\left\{W_{n}\right\}_{n=0}^{\infty}$ by $W_{0} \stackrel{\text { df }}{=} I$ and $W_{n} \stackrel{\text { df }}{=}$ $U_{n-1} U_{n-2} \ldots U_{0}$ for $n \geqslant 1$. An easy calculation shows that $U_{+} U=W U_{+} W^{*}$, and so we have

$$
T=U_{+} U S=W U_{+} W^{*} S=W\left(U_{+} W^{*} S W\right) W^{*} .
$$

Hence $T$ is unitarily equivalent to $U_{+} W^{*} S W$. Since $U_{+} W^{*} S W$ is the operator valued weighted shift with weights $\left\{W_{n}^{*} S_{n} W_{n}\right\}_{n=0}^{\infty}$, by the definitions of $W$ and $S_{n},(2.2)$ and (2.1) we obtain

$$
\begin{aligned}
W_{n}^{*} S_{n} W_{n} W_{n-1}^{*} S_{n-1} W_{n-1} \ldots W_{1}^{*} S_{1} W_{1} W_{0}^{*} S_{0} W_{0} & =\left(U_{n-1} \ldots U_{0}\right)^{*} S_{n} U_{n-1} S_{n-1} \ldots U_{0} S_{0} \\
& =V_{n}^{*} T_{n} U_{n-1} S_{n-1} \ldots U_{0} S_{0}=P_{n}
\end{aligned}
$$

for all $n \geqslant 0$; that is, $U_{+} W^{*} S W \in l_{I W P}^{2}(\mathcal{H})$.

COROLlary 2.3. If $T \in l_{I W}^{2}(\mathcal{H})$ is a 2-isometric (resp. completely hyperexpansive, $k$-hyperexpansive) operator valued weighted shift, then $T$ is unitarily equivalent to a 2isometric (resp. completely hyperexpansive, $k$-hyperexpansive) operator valued weighted shift $T^{\prime} \in l_{I W P}^{2}(\mathcal{H})$.

REMARK 2.4. Let $T \in \boldsymbol{B}\left(l^{2}(\mathcal{H})\right)$ be an operator valued weighted shift with weights $\left\{T_{n}\right\}_{n=0}^{\infty}$ satisfying $\overline{\mathcal{R}\left(T_{n}\right)}=\mathcal{H}$, for all $n \geqslant 0$, and suppose that there exists $i_{0} \geqslant 0$ such that $T_{i_{0}}$ is not injective. Then $T$ is not unitarily equivalent to any operator valued weighted shift $T^{\prime}$ with weights $\left\{T_{n}^{\prime}\right\}_{n=0}^{\infty}$ satisfying $T_{n}^{\prime} \ldots T_{0}^{\prime}$ are positive (even selfadjoint) for all $n \geqslant 0$. Indeed, suppose contrary to our claim, that such an operator $T^{\prime}$ exists. An easy calculation shows that $\overline{\mathcal{R}\left(T_{n}^{\prime}\right)}=\mathcal{H}$ for all $n \geqslant 0$ and $\overline{\mathcal{R}\left(T_{0}^{\prime} \ldots T_{i_{0}}^{\prime}\right)}=\mathcal{H}$. Consequently, $T_{0}^{\prime} \ldots T_{i_{0}}^{\prime}$ is injective. But, by Lemma 2.1 , there exist unitary operators $U_{0}$ and $U_{i_{0}+1}$ such that $U_{i_{0}+1} T_{0} \ldots T_{i_{0}}=T_{0}^{\prime} \ldots T_{i_{0}}^{\prime} U_{0}$, which is impossible because $T_{i_{0}}$ is not injective. In the same way we can prove that $T$ is not unitarily equivalent to any operator valued weighted shift with positive (or self-adjoint) weights.

For an operator valued unilateral weighted shift $T$ with weights $\left\{T_{n}\right\}_{n=0}^{\infty}$ in $\mathcal{H}$ we set

$$
T_{[m, n]} \stackrel{\text { df }}{=} \begin{cases}T_{m-1} T_{m-2} \cdots T_{n} & \text { if } m>n \\ I & \text { if } m=n\end{cases}
$$

Let us define

$$
\Delta_{k, s}(f) \stackrel{\mathrm{df}}{=} \sum_{0 \leq p \leq k}(-1)^{p}\left(\begin{array}{l}
k \\
p
\end{array}\right)\left\|T_{[s+p, s]} f\right\|^{2} \quad(k \geqslant 1, s \geqslant 0) .
$$

Since the proof of the following fact is similar to the proof of [4, Lemma 6.1] and [5, Proposition 1.3], we leave it to the reader.

Proposition 2.5. Let $T$ be an operator valued unilateral weighted shift with weights $\left\{T_{n}\right\}_{n=0}^{\infty}$ and fix $k \geqslant 1$.

(i) $T$ is a $k$-isometry if and only if $\Delta_{k, s}(f)=0$, for all $s \geqslant 0$ and $f \in \mathcal{H}$. 
(ii) $T$ is $k$-expansive if and only if $\Delta_{k, s}(f) \leq 0$, for all $s \geqslant 0$ and $f \in \mathcal{H}$.

(iii) $T$ is $k$-hyperexpansive if and only if $\Delta_{n, s}(f) \leq 0$, for all $s \geqslant 0, f \in \mathcal{H}$ and $n=$ $1, \ldots, k$.

(iv) $T$ is completely hyperexpansive if and only if $\Delta_{n, s}(f) \leq 0$, for all $s \geqslant 0, f \in \mathcal{H}$ and $n \geqslant 1$.

Moreover, if all operators $T_{i}$, where $i \geqslant 0$, are invertible, then the statement " $s \geqslant 0$ " in condition (iv) can be replaced by " $s=0$ ".

PROPOSITION 2.6. If $T$ is a 2-expansive operator valued unilateral weighted shift with weights $\left\{T_{n}\right\}_{n=0}^{\infty}$, then $T$ leaves its domain invariant.

Proof. Take a vector $f \in \mathcal{D}(T)$. Then $f=\oplus_{n=0}^{\infty} f_{n}$ with $f_{n} \in \mathcal{H}$ and $\sum_{n=0}^{\infty}\left\|T_{n} f_{n}\right\|^{2}<$ $\infty$. Since $T$ is 2 -expansive and

$$
g_{n} \stackrel{\text { df }}{=} \stackrel{(0)}{0} \oplus \stackrel{(1)}{0} \oplus \ldots \oplus \stackrel{(n-1)}{0} \oplus \stackrel{(n)}{f_{n}} \oplus \stackrel{(n+1)}{0} \oplus \ldots \in \mathcal{D}\left(T^{2}\right),
$$

it follows, by Proposition 2.5 (ii), that $\left\|T_{n+1} T_{n} f_{n}\right\|^{2} \leq 2\left\|T_{n} f_{n}\right\|^{2}$, for every $n \geqslant 0$. Consequently

$$
\sum_{n=0}^{\infty}\left\|T_{n+1}\left(T_{n} f_{n}\right)\right\|^{2} \leq 2 \sum_{n=0}^{\infty}\left\|T_{n} f_{n}\right\|^{2}<\infty
$$

which yields $T f \in \mathcal{D}(T)$.

3. 2-isometric operator valued unilateral weighted shifts. In this section we intend to characterize 2-isometric invertibly weighted shifts. We start with the following result.

Lemma 3.1. If the operators $A \in \boldsymbol{B}(\mathcal{H})$ and $C \in \boldsymbol{B}(\mathcal{H} \ominus \mathcal{R}(A))$ satisfy $A^{*} A \geqslant I_{\mathcal{H}}$ and $C^{*} C \geqslant I_{\mathcal{H} \ominus \mathcal{R}(A)}$, respectively, then there exists a positive and invertible operator $D \in \boldsymbol{B}(\mathcal{R}(A))$ such that the operator $B \stackrel{\mathrm{df}}{=} C \oplus D$ satisfies $B^{*} B \geqslant I_{\mathcal{H}}$ and

$$
\|B A f\|^{2}=2\|A f\|^{2}-\|f\|^{2}, \quad f \in \mathcal{H} .
$$

Moreover, if $A$ and $B$ are invertible and an invertible operator $B_{1} \in \boldsymbol{B}(\mathcal{H})$ satisfies

$$
\left\|B_{1} A f\right\|^{2}=2\|A f\|^{2}-\|f\|^{2}, \quad f \in \mathcal{H},
$$

then there exists a unitary operator $U \in \boldsymbol{B}(\mathcal{H})$ such that $B_{1}=U B$.

Proof. Since $\mathcal{R}(A)$ is closed and $\operatorname{dim} \mathcal{R}(A)=\operatorname{dim} \mathcal{H}$, it follows that there exists a unitary operator $U: \mathcal{H} \rightarrow \mathcal{R}(A)$. Set $S \stackrel{\text { df }}{=} U A^{-1} \in \boldsymbol{B}(\mathcal{R}(A))$, where $A^{-1}$ is considered as the operator from $\mathcal{R}(A)$ to $\mathcal{H}$. Notice that $\left\|A^{-1} f\right\|=\||S| f\|$, where $|S| \stackrel{\text { df }}{=}\left(S^{*} S\right)^{\frac{1}{2}}$. Since $|S|$ is a positive contraction, there exists a spectral measure $E$ on $\mathcal{R}(A)$ such that $|S|=$ $\int_{[0,1]} x E(\mathrm{~d} x)$. Let us define the operator $D \in \boldsymbol{B}(\mathcal{R}(A))$ by $D=\left(\int_{[0,1]} 2-x^{2} E(\mathrm{~d} x)\right)^{\frac{1}{2}}$. It is clear that $\|f\| \leq\|D f\|$. Hence $D$ is invertible and $\|f\| \leq\|B f\|$, for all $f \in \mathcal{H}$. An easy computation shows that

$$
\left\|\left(\int_{[0,1]} 2-x^{2} E(\mathrm{~d} x)\right)^{\frac{1}{2}} A f\right\|^{2}=2\|A f\|^{2}-\||S| A f\|^{2}=2\|A f\|^{2}-\|f\|^{2} \quad(f \in \mathcal{H}),
$$

and so (3.1) holds. 
If $A$ and $B$ are invertible operators and an invertible operator $B_{1}$ satisfies (3.2), then $\left\|B_{1} f\right\|=\|B f\|$, for all $f \in \mathcal{H}$. Hence $U B f=B_{1} f$, for $f \in \mathcal{H}$, defines a unitary operator $U$.

Proposition 3.2. Let $T_{0} \in \boldsymbol{B}(\mathcal{H})$ be an operator (resp. an invertible operator) such that $T_{0}^{*} T_{0} \geqslant I_{\mathcal{H}}$. Then there exists a family of operators (resp. invertible operators) $\left\{T_{i}\right\}_{i=1}^{\infty}$ such that the operator valued weighted shift with weights $\left\{T_{i}\right\}_{i=0}^{\infty}$ is a 2-isometry.

Proof. Existence of such a sequence $\left\{T_{i}\right\}_{i=1}^{\infty}$ is an inductive consequence of Proposition 2.5 (i), Lemma 3.1 and Proposition 2.2.

It has been shown in [4, Lemma 6.1] that the (scalar) weighted shift $S$ with weights $\left\{\lambda_{n}\right\}_{n=0}^{\infty}$ is a 2-isometry if and only if $\lambda_{0} \geqslant 1$ and $\lambda_{n}=\sigma_{n}\left(\lambda_{0}\right)$ for $n \geqslant 0$, where

$$
\sigma_{n}(\lambda)=\sqrt{\frac{1+(n+1)\left(\lambda^{2}-1\right)}{1+n\left(\lambda^{2}-1\right)}} \quad(n \geqslant 0, \lambda \geqslant 1) .
$$

In the case of operator valued weighted shifts we have the following result.

THEOREM 3.3. If $T_{0} \geqslant I$, then there exists exactly one sequence $\left\{T_{i}\right\}_{i=1}^{\infty}$ such that $\left\{T_{n}\right\}_{n=1}^{\infty}$ completes $T_{0}$ to the weights of a 2-isometric operator $T \in l_{I W P}^{2}(\mathcal{H})$. Moreover, the sequence $\left\{T_{n}\right\}_{n=0}^{\infty}$ is composed of positive and commuting operators.

Proof. If $T_{0} \geqslant I$, then there exists a spectral measure $E$ such that $T_{0}=$ $\int_{\left[1,\left\|T_{0}\right\|\right]} x E(\mathrm{~d} x)$. Suppose that $T_{0}, \ldots, T_{n}$ are defined and $T_{n}=\int_{\left[1,\left\|T_{0}\right\|\right]} \xi(x) E(\mathrm{~d} x)$, where $\xi(x) \geqslant 1$ for $x \in\left[1,\left\|T_{0}\right\|\right]$. Set

$$
T_{n+1} \stackrel{\mathrm{df}}{=} \int_{\left[1,\left\|T_{0}\right\|\right]} \sqrt{\frac{2 \xi^{2}(x)-1}{\xi^{2}(x)}} E(\mathrm{~d} x) .
$$

Then

$$
\sqrt{\frac{2 \xi^{2}(x)-1}{\xi^{2}(x)}} \geqslant 1, \quad x \in\left[1,\left\|T_{0}\right\|\right],
$$

and $\left\|T_{n+1} T_{n} f\right\|^{2}=2\left\|T_{n} f\right\|^{2}-\|f\|^{2}$ for $f \in \mathcal{H}$.

Suppose that families $\left\{T_{i}\right\}_{i=1}^{\infty}$ and $\left\{T_{i}^{\prime}\right\}_{i=1}^{\infty}$ complete $T_{0}$ to the weights of 2-isometric weighted shifts $T \in l_{I W P}^{2}(\mathcal{H})$ and $T^{\prime} \in l_{I W P}^{2}(\mathcal{H})$ respectively. An easy computation shows that, if an operator $T$ is a 2-isometry, then $T$ is a $k$-isometry for $k \geqslant 2$. Suppose that $T_{i}^{\prime} \cdots T_{1}^{\prime} T_{0}=T_{i} \cdots T_{1} T_{0}$ for $i=0, \ldots, k-1$. Since $T$ and $T^{\prime}$ are $(k+1)$ isometries, we infer from Proposition 2.5 (i) that

$$
\begin{aligned}
& \left\|T_{k}^{\prime} \cdots T_{1}^{\prime} T_{0} f\right\|^{2}=(-1)^{k+1} \sum_{0 \leq p \leq k}(-1)^{p}\left(\begin{array}{c}
k+1 \\
p
\end{array}\right)\left\|T_{[p, 0]}^{\prime} f\right\|^{2} \\
& =(-1)^{k+1} \sum_{0 \leq p \leq k}(-1)^{p}\left(\begin{array}{c}
k+1 \\
p
\end{array}\right)\left\|T_{[p, 0]} f\right\|^{2}=\left\|T_{k} \cdots T_{1} T_{0} f\right\|^{2} \quad(f \in \mathcal{H}) .
\end{aligned}
$$

Since $T_{k} \cdots T_{1} T_{0}$ and $T_{k}^{\prime} \cdots T_{1}^{\prime} T_{0}$ are positive, we have $T_{k}^{\prime} \cdots T_{1}^{\prime} T_{0}=T_{k} \cdots T_{1} T_{0}$. 
REMARK 3.4. It follows from [9, Lemma 1] that if $T$ is a 2-isometric operator valued weighted shift then $T_{0}^{*} T_{0} \geqslant I$. Notice that, by Proposition 2.5 (i), if $T$ is a 2-isometric invertibly weighted shift, then $T$ is a bounded operator.

4. Completely hyperexpansive operator valued unilateral weighted shifts. In this section we investigate the completely hyperexpansive invertibly weighted shifts. There is no loss of generality in assuming that the considered weighted shifts belong to the class $l_{I W P}^{2}(\mathcal{H})($ cf. Corollary 2.3).

For a semispectral measure $F: \mathcal{B}([0,1]) \longrightarrow \boldsymbol{B}(\mathcal{H})$ we set

$$
\begin{aligned}
& \Omega_{F, 0} \stackrel{\mathrm{df}}{=} I_{\mathcal{H}}, \\
& \Omega_{F, n} \stackrel{\mathrm{df}}{=}\left(I+\int_{[0,1]}\left(1+\ldots+x^{n-1}\right) F(\mathrm{~d} x)\right)^{\frac{1}{2}} \quad(n \geqslant 1) .
\end{aligned}
$$

Proposition 4.1. Let $T \in \boldsymbol{B}\left(l^{2}(\mathcal{H})\right)$ be the operator valued weighted shift with weights $\left\{T_{n}\right\}_{n=0}^{\infty}$. Then $T$ is completely hyperexpansive and $T \in l_{I W P}^{2}(\mathcal{H})$ if and only if there exists a semispectral measure $F: \mathcal{B}([0,1]) \longrightarrow \boldsymbol{B}(\mathcal{H})$ such that

$$
\begin{gathered}
T_{0}=\Omega_{F, 1}, \\
T_{n}=\Omega_{F, n+1} \Omega_{F, n}^{-1}=\Omega_{F, n+1} T_{0}^{-1} T_{1}^{-1} \cdots T_{n-1}^{-1} \quad(n \geqslant 1) .
\end{gathered}
$$

The measure $F$ is uniquely determined by the condition

$$
\|f\|^{2}+\int_{[0,1]}\left(1+\cdots+x^{k}\right)\langle F(\mathrm{~d} x) f, f\rangle=\left\langle T_{0}^{*} \ldots T_{k}^{*} T_{k} \ldots T_{0} f, f\right\rangle \quad(f \in \mathcal{H}, k \geqslant 0) .
$$

Moreover, $\left\{T_{n}\right\}_{n=0}^{\infty}$ is a commutative family of operators if and only if $F$ is commutative.

Proof. Let $T \in l_{I W P}^{2}(\mathcal{H})$ be completely hyperexpansive. Since

$$
T_{0}^{*} \ldots T_{n}^{*} T_{n} \ldots T_{0}=\left(T_{n} \ldots T_{0}\right)^{2} \quad(n \geqslant 0),
$$

and the sequence $I, T_{0}^{2},\left(T_{1} T_{0}\right)^{2}, \ldots$ is completely alternating (cf. [1, Remark 2]), it follows from Theorem 1.2 that there exists a semispectral measure $F$ on $[0,1]$ such that

$$
\left(T_{0} \ldots T_{n}\right)^{2}=I+\int_{[0,1]}\left(1+\cdots+x^{n}\right) F(\mathrm{~d} x) \quad(n \geqslant 0) .
$$

The reverse implication follows from Proposition 2.5.

Suppose that $\left\{T_{n}\right\}_{n=0}^{\infty}$ is a commutative family of operators. Then, by the equality $\left(T_{m} \ldots T_{0}\right)^{2}\left(T_{n} \ldots T_{0}\right)^{2}=\left(T_{n} \ldots T_{0}\right)^{2}\left(T_{m} \ldots T_{0}\right)^{2}$, for $m, n \geqslant 0$, we get

$$
\Omega_{F, m}^{2} \Omega_{F, n}^{2}=\Omega_{F, n}^{2} \Omega_{F, m}^{2} \quad m, n \geqslant 1 .
$$

Using an induction argument we obtain

$$
\int_{[0,1]} x^{m} F(\mathrm{~d} x) \int_{[0,1]} x^{n} F(\mathrm{~d} x)=\int_{[0,1]} x^{n} F(\mathrm{~d} x) \int_{[0,1]} x^{m} F(\mathrm{~d} x) \quad(m, n \geqslant 0) .
$$


Hence, by the Stone-Weierstrass approximation theorem $F(\sigma) F(\tau)=F(\tau) F(\sigma)$, for all closed subsets $\sigma, \tau \subseteq[0,1]$, which together with the (automatic) regularity of $F$ implies that $F$ is commutative. The reverse implication follows from [2, Theorem 14].

We are now in a position to show how the minimal objects introduced in [5] look in the context of bounded completely hyperexpansive operator valued weighted shifts $T \in l_{I W P}^{2}(\mathcal{H})$.

Take a semispectral measure $F$ on $[0,1]$ and let $(\tilde{\mathcal{K}}, \tilde{R}, \tilde{E})$ be a minimal spectral dilation of $F$. Let $T=S_{F}$ be a weighted shift defined in Proposition 4.1. Set

$$
\begin{aligned}
& F_{n}(\sigma) \stackrel{\text { df }}{=} \Omega_{F, n}^{-1} \int_{\sigma} x^{n} F(\mathrm{~d} x) \Omega_{F, n}^{-1} \quad(n \geqslant 0, \sigma \in \mathcal{B}([0,1])), \\
& \mathcal{K}_{n} \stackrel{\text { df }}{=} \bigvee_{i \geqslant 0} \int_{[0,1]} x^{i+\frac{n}{2}} \tilde{E}(\mathrm{~d} x) \tilde{R}(\mathcal{H}) \quad(n \geqslant 0),
\end{aligned}
$$

and define a semispectral measure $G: \mathcal{B}([0,1]) \rightarrow \boldsymbol{B}\left(l^{2}(\mathcal{H})\right)$, a Hilbert space $\mathcal{K}$, an operator $R \in \boldsymbol{B}\left(l^{2}(\mathcal{H}), \mathcal{K}\right)$, a spectral measure $E: \mathcal{B}([0,1]) \rightarrow \boldsymbol{B}(\mathcal{K})$ and an operator $A \in \boldsymbol{B}(\mathcal{K})$ by

(i) $G(\sigma)\left(\oplus_{i=0}^{\infty} f_{i}\right) \stackrel{\text { df }}{=} \oplus_{i=0}^{\infty} F_{i}(\sigma) f_{i} \quad\left(\sigma \in \mathcal{B}([0,1]), \oplus_{i=0}^{\infty} f_{i} \in l^{2}(\mathcal{H})\right)$,

(ii) $\mathcal{K} \stackrel{\text { df }}{=} \oplus_{i=0}^{\infty} \mathcal{K}_{i}$,

(iii) $R \stackrel{\mathrm{df}}{=} \oplus_{i=0}^{\infty}\left[P_{\mathcal{K}_{i}} \int_{[0,1]} x^{\frac{i}{2}} \tilde{E}(\mathrm{~d} x) \tilde{R} \Omega_{F, i}^{-1}\right]$

(iv) $\left.E(\sigma)\left(\oplus_{i=0}^{\infty} f_{i}\right) \stackrel{\mathrm{df}}{=} \bigoplus_{i=0}^{\infty} P_{\mathcal{K}_{i}} \tilde{E}(\sigma)\right|_{\mathcal{K}_{i}} f_{i} \quad\left(\sigma \in \mathcal{B}([0,1]), \oplus_{i=0}^{\infty} f_{i} \in \mathcal{K}\right)$,

(v) $A \stackrel{\text { df }}{=} \oplus_{i=0}^{\infty}\left[\left.P_{\mathcal{K}_{i}} \int_{[0,1]} x \tilde{E}\right|_{\mathcal{K}_{i}}(\mathrm{~d} x)\right]$,

where $P_{\mathcal{K}_{i}} \in \boldsymbol{B}\left(\mathcal{K}, \mathcal{K}_{i}\right)$ is the orthogonal projection of $\mathcal{K}$ onto $\mathcal{K}_{i}$.

THEOREM 4.2. $G$ is a semispectral measure satisfying $(1.1),(\mathcal{K}, R, E)$ is a minimal spectral dilation of $F,(\mathcal{K}, R, A)$ is a CH-minimal spectral decomposition of $T$ and $\sigma(A)=$ $\operatorname{supp} G=\operatorname{supp} F$. Moreover, $L^{1}(G)=L^{1}(F)$ and $\|f\|_{1, G}=\|f\|_{1, F}$ for each $f \in L^{1}(G)$ (cf. [11, Appendix)].

Proof. From the equations

$$
\begin{aligned}
& \left\langle\int_{[0,1]} x^{n} G(\mathrm{~d} x) \oplus_{i=0}^{\infty} f_{i}, \oplus_{j=0}^{\infty} g_{i}\right\rangle \\
& \quad=\int_{[0,1]} x^{n}\left\langle G(\mathrm{~d} x) \oplus_{i=0}^{\infty} f_{i}, \oplus_{j=0}^{\infty} g_{j}\right\rangle \\
& \quad=\sum_{i=0}^{\infty} \int_{[0,1]} x^{n}\left\langle F_{i}(\mathrm{~d} x) f_{i}, g_{i}\right\rangle \\
& \quad=\sum_{i=0}^{\infty}\left\langle\int_{[0,1]} x^{n+i} F(\mathrm{~d} x) \Omega_{F, i}^{-1} f_{i}, \Omega_{F, i}^{-1} g_{i}\right\rangle \\
& \quad=\left\langle\oplus_{i=0}^{\infty}\left[\Omega_{F, i}^{-1} \int_{[0,1]} x^{n+i} F(\mathrm{~d} x) \Omega_{F, i}^{-1}\right] \oplus_{i=0}^{\infty} f_{i}, \oplus_{j=0}^{\infty} g_{i}\right\rangle \quad(n \geqslant 0),
\end{aligned}
$$

and Proposition 4.1, we see that

$$
\int_{[0,1]} x^{n} G(\mathrm{~d} x)=\oplus_{i=0}^{\infty} \Omega_{F, i}^{-1} \int_{[0,1]} x^{n+i} F(\mathrm{~d} x) \Omega_{F, i}^{-1}=T^{*(n+1)} T^{n+1}-T^{* n} T^{n} \quad(n \geqslant 0) .
$$


Hence

$$
\begin{aligned}
T^{* n} T^{n} & =\left(T^{*(n)} T^{n}-T^{*(n-1)} T^{n-1}\right)+\cdots+\left(T^{*} T-I\right)+I \\
& =I+\int_{[0,1]}\left(1+\cdots+x^{n-1}\right) G(\mathrm{~d} x) \quad(n \geqslant 1),
\end{aligned}
$$

and so (1.1) holds. Note that, by the definition of $F_{i}$ and [5, Theorem 4.4], we have $\sigma(A)=\operatorname{supp} G=\operatorname{supp} F$. It follows from the definition of $F_{i}$ that

$$
\begin{aligned}
\int_{[0,1]} x^{n} F_{i}(\mathrm{~d} x) & =\Omega_{F, i}^{-1} \int_{[0,1]} x^{i+n} F(\mathrm{~d} x) \Omega_{F, i}^{-1} \\
& =\Omega_{F, i}^{-1} \tilde{R}^{*} \int_{[0,1]} x^{\frac{i}{2}} \tilde{E}(\mathrm{~d} x) \int_{[0,1]} x^{n} \tilde{E}(\mathrm{~d} x) \int_{[0,1]} x^{\frac{i}{2}} \tilde{E}(\mathrm{~d} x) \tilde{R} \Omega_{F, i}^{-1} \quad(n \geqslant 0),
\end{aligned}
$$

and so $(\mathcal{K}, R, E)$ is a minimal spectral dilation of $G$. It is easy to check that $(\mathcal{K}, R, A)$ is a $\mathrm{CH}$-minimal spectral decomposition of $T$. Take $\xi \in L^{1}(G)$. Since

$$
\int_{[0,1]}|\xi(x)|\left\langle F_{i}(\mathrm{~d} x) f, f\right\rangle=\int_{[0,1]}|\xi(x)| x^{i}\left\langle F(\mathrm{~d} x) \Omega_{F, i}^{-1} f, \Omega_{F, i}^{-1} f\right\rangle \leq\|f\|^{2}\|\xi\|_{1, F},
$$

for all $f \in \mathcal{H}$, we see that

$$
\begin{aligned}
& \int_{[0,1]}|\xi(x)|\left|G(\mathrm{~d} x) \oplus_{i=0}^{\infty} f_{i}, \oplus_{i=0}^{\infty} f_{i}\right\rangle \\
& \quad=\sum_{i=0}^{\infty} \int_{[0,1]}|\xi(x)|\left\langle F_{i}(\mathrm{~d} x) f_{i}, f_{i}\right\rangle \leq\|\xi\|_{1, F}\left\|\oplus_{i=0}^{\infty} f_{i}\right\|^{2} \quad\left(\oplus_{i=0}^{\infty} f_{i} \in \mathcal{K}\right) .
\end{aligned}
$$

Consequently $L^{1}(G)=L^{1}(F)$ and $\|\xi\|_{1, G}^{2}=\|\xi\|_{1, F}^{2}$.

We shall now consider the problem of a completely hyperexpansive completion of positive operators $T_{0}, T_{1} \in \boldsymbol{B}(\mathcal{H})$. The similar problem for subnormal operators has been considered by Ivanovski in [3] (cf. [10]).

THEOREM 4.3. Let $T_{0}$ and $T_{1}$ be commuting positive operators. Then the following are equivalent.

(i) There exist weights $\left\{T_{n}\right\}_{n=2}^{\infty}$ such that the operator valued unilateral weighted shift $T \in \boldsymbol{B}\left(l^{2}(\mathcal{H})\right)$ with weights $\left\{T_{n}\right\}_{n=0}^{\infty}$ is completely hyperexpansive and $T \in l_{I W P}^{2}(\mathcal{H})$.

(ii) $T_{1} \geqslant I$ and $\left(T_{1} T_{0}\right)^{2}-2 T_{0}^{2}+I \leq 0$.

Proof. (ii) $\Rightarrow$ (i). Suppose that $T_{1} \geqslant I$ and $\left(T_{1} T_{0}\right)^{2}-2 T_{0}^{2}+I \leq 0$. Let us define the operators $A, B \in \boldsymbol{B}(\mathcal{H})$ by $A=-\left(T_{1} T_{0}\right)^{2}+2 T_{0}^{2}-I$ and $B=\left(T_{1} T_{0}\right)^{2}-T_{0}^{2}$. By assumption $A$ and $B$ are positive. Let us define a semispectral measure $F: \mathcal{B}([0,1]) \longrightarrow$ $\boldsymbol{B}(\mathcal{H})$ by $F(\sigma) \stackrel{\text { df }}{=} \chi_{0} A+\chi_{1} B, \sigma \in \mathcal{B}([0,1])$, where $\chi_{\sigma}$ stands for the characteristic function of the set $\sigma$. Let $S_{F}=\left\{\left(S_{F}\right)_{n}\right\}_{n=0}^{\infty}$ be a completely hyperexpansive weighted shift defined as in Proposition 4.1. It follows from (4.1) that $\left(S_{F}\right)_{0}=T_{0}$ and $\left(S_{F}\right)_{1}=T_{1}$.

The implication (i) $\Rightarrow$ (ii) is obvious.

5. Examples. This section contains various examples illustrating the subject of the paper. We begin with examples of 2-isometric weighted shifts and completely 
hyperexpansive weighted shifts which are not 2-isometric. Both cases, commutative and noncommutative weights, are taken into consideration.

EXAMPLE 5.1. Let $\mathcal{H} \stackrel{\text { df }}{=} L^{2}([1,2], m)$, where $m$ stands for the Lebesgue measure on $[1,2]$. Let us define the spectral measure $E: \mathcal{B}([1,2]) \longrightarrow \boldsymbol{B}(\mathcal{H})$ by $E(\sigma) f \stackrel{\text { df }}{=} \chi_{\sigma} f$ for $\sigma \in \mathcal{B}([1,2])$ and $f \in L^{2}([1,2], m)$, where $\chi_{\sigma}$ stands for the characteristic function of the set $\sigma$. Set $T_{0}=\int_{[1,2]} x^{\frac{1}{2}} E(\mathrm{~d} x)$. Then, by Theorem 3.3, $T_{0}$ can be completed to a 2isometric weighted shift $T \in l_{I W P}^{2}(\mathcal{H})$ that is not unitarily equivalent to any orthogonal sum of scalar weighted shifts $\oplus_{i=0}^{\infty} S_{i}$. Indeed, suppose contrary to our claim, that $T$ is unitarily equivalent to $\oplus_{i=0}^{\infty} S_{i}$, which in turn is unitarily equivalent to an operator valued weighted shift $T^{\prime}$ with diagonal weights. By [7, Corollary 3.3] there exists a unitary operator $U$ on $\mathcal{H}$ such that $U T_{0}^{* *} T_{0}^{\prime}=T_{0}^{*} T_{0} U=\int_{[1,2]} x E(\mathrm{~d} x) U$, which is impossible, because the point spectrum of $\int_{[1,2]} x E(\mathrm{~d} x)$ is an empty set, but the point spectrum of $T_{0}^{*} T_{0}^{\prime}$ is not empty.

Let us define the semispectral measure $F: \mathcal{B}([1,2]) \longrightarrow \boldsymbol{B}(\mathcal{H})$ by $F(\sigma) \stackrel{\text { df }}{=} m(\sigma) T_{0}$ $(\sigma \in \mathcal{B}([1,2]))$. A similar calculation shows that an operator $S_{F}$, defined as in Proposition 4.1, is a completely hyperexpansive operator with commutative weights that is not unitarily equivalent to any orthogonal sum of scalar weighted shifts $\oplus_{i=0}^{\infty} S_{i}$. An easy calculation shows that $S_{F}$ is not a 2 -isometry.

Let $A, B \in \boldsymbol{B}(\mathcal{H})$ be two positive non-commuting operators and define a semispectral measure $G: \mathcal{B}([0,1]) \longrightarrow \boldsymbol{B}(\mathcal{H})$ by $G(\sigma) \stackrel{\text { df }}{=} \chi_{\{0\}} A+\chi_{\{1\}} B$. Then an operator $S_{G}$ defined in Proposition 4.1 is a completely hyperexpansive operator with noncommutative weights which, by Theorem 3.3 , is not a 2 -isometry.

In Example 5.2, we construct two completely hyperexpansive weighted shifts $T, T^{\prime} \in l_{I W P}^{2}(\mathbb{C})$ satisfying $T \neq T^{\prime}, T_{0}=T_{0}^{\prime}$ and $T_{1}=T_{1}^{\prime}$ (cf. Theorem 4.3).

EXAMPLE 5.2. Let us define the measures $\mu_{1}$ and $\mu_{2}$ on $[0,1]$ by $\mu_{1}=2 \chi_{\left\{\frac{1}{2}\right\}}$ and $\mu_{2}=\chi_{\left\{\frac{1}{3}\right\}}+\chi_{\left\{\frac{2}{3}\right\}}$. Then the operators $S_{\mu_{1}}$ and $S_{\mu_{2}}$ defined as in Proposition 4.1 satisfy $S_{\mu_{1}} \neq S_{\mu_{2}},\left(S_{\mu_{1}}\right)_{0}=\left(S_{\mu_{2}}\right)_{0}$ and $\left(S_{\mu_{1}}\right)_{1}=\left(S_{\mu_{2}}\right)_{1}$.

In Example 5.3 we show that the condition

$$
\sum_{0 \leq p \leq n}(-1)^{p}\left(\begin{array}{l}
n \\
p
\end{array}\right)\left\|T_{[p, 0]} f\right\|^{2}=0 \quad(f \in \mathcal{H}, n \geqslant 1)
$$

does not imply complete hyperexpansivity in the case in which $T$ is not an invertible weighted shift (cf. Proposition 2.5).

EXAMPLE 5.3. Let $\mathcal{H}$ be an infinite dimensional Hilbert space and $U \in \boldsymbol{B}(\mathcal{H} \oplus \mathcal{H})$ an isometric operator such that $\mathcal{R}(U)=\mathcal{H} \oplus 0$. Let us consider the operator weighted shift $T \in \boldsymbol{B}(\mathcal{H} \oplus \mathcal{H})$, where $T_{0}=U, T_{1}=I_{\mathcal{H}} \oplus\left(\frac{1}{2} I_{\mathcal{H}}\right)$ and $T_{n}=I_{\mathcal{H} \oplus \mathcal{H}}$, for all $n \geqslant 2$. An easy calculation shows that $T$ satisfies (5.1). Since $\left\|T_{1}(0 \oplus f)\right\|=\frac{1}{2}\|f\|$ for $f \in \mathcal{H}$, it follows that $T$ is not completely hyperexpansive (cf. [9, Lemma 1]).

We conclude the paper with an example of a 2-isometric unbounded operator valued weighted shift. 
EXAMPLE 5.4. Let $\mathcal{K}_{00}$ and $\mathcal{K}_{01}$ be separable infinite dimensional Hilbert spaces and set $\mathcal{H} \stackrel{\text { df }}{=} \mathcal{K}_{00} \oplus \mathcal{K}_{01}$.

Step 1. Suppose that $\mathcal{K}_{01}=\mathcal{K}_{11} \oplus \mathcal{K}_{12} \oplus \mathcal{K}_{13}$, where $\mathcal{K}_{11}, \mathcal{K}_{12}$ and $\mathcal{K}_{13}$ are closed infinite dimensional subspaces of $\mathcal{K}_{01}$. Let $A_{00} \in \boldsymbol{B}\left(\mathcal{K}_{00}\right)$ be an operator such that $A_{00} \geqslant I$ and $\left\|A_{00}\right\| \geqslant 2$, and let $V_{01} \in \boldsymbol{B}\left(\mathcal{K}_{01}\right)$ be an isometry such that $\mathcal{R}\left(V_{01}\right)=\mathcal{K}_{11}$. We can now define the weight $T_{0}$ on $\mathcal{H}$ by $T_{0} \stackrel{\text { df }}{=} A_{00} \oplus V_{01}$. Set $\mathcal{K}_{n 0} \stackrel{\text { df }}{=} \mathcal{K}_{00}$, for $n \geqslant 1$, and $\mathcal{K}_{n 1} \stackrel{\text { df }}{=} \mathcal{K}_{11}$, for $n \geqslant 2$. It follows from Theorem 3.3 that there exist weights $\left\{A_{n 0}\right\}_{n=1}^{\infty}$ on $\mathcal{K}_{00}$ such that the weighted shift with weights $\left\{A_{n 0}\right\}_{n=0}^{\infty}$ is a 2 -isometry. Define $V_{n 1} \stackrel{\text { df }}{=} I_{\mathcal{K}_{11}}$ for $n \geqslant 1$.

Step 2. Suppose that $\mathcal{K}_{13}=\mathcal{K}_{23} \oplus \mathcal{K}_{24} \oplus \mathcal{K}_{25}$, where $\mathcal{K}_{23}, \mathcal{K}_{24}$ and $\mathcal{K}_{25}$ are closed infinite dimensional subspaces of $\mathcal{K}_{13}$. Let $A_{12} \in \boldsymbol{B}\left(\mathcal{K}_{12}\right)$ be an operator such that $A_{12} \geqslant I$ and $\left\|A_{12}\right\| \geqslant 3$. Let $V_{13} \in \boldsymbol{B}\left(\mathcal{K}_{13}\right)$ be an isometry such that $\mathcal{R}\left(V_{13}\right)=\mathcal{K}_{23}$. We can now define the weight $T_{1}$ on $\mathcal{H}$ by $T_{1} \stackrel{\text { df }}{=} A_{10} \oplus V_{11} \oplus A_{12} \oplus V_{13}$. Set $\mathcal{K}_{n 2} \stackrel{\text { df }}{=} \mathcal{K}_{12}$ for $n \geqslant 2$ and $\mathcal{K}_{n 3} \stackrel{\mathrm{df}}{=} \mathcal{K}_{23}$ for $n \geqslant 3$. It follows from Theorem 3.3 that there exist weights $\left\{A_{n 2}\right\}_{n=2}^{\infty}$ on $\mathcal{K}_{00}$ such that the weighted shift with weights $\left\{A_{n 2}\right\}_{n=1}^{\infty}$ is a 2-isometry. Define $V_{n 3} \stackrel{\text { df }}{=} I_{\mathcal{K}_{23}}$ for $n \geqslant 2$.

Using this and an induction argument we can construct a sequence of bounded operators $\left\{T_{n}\right\}_{n=0}^{\infty}$ defined on $\mathcal{H}$, where $\left\|T_{n}\right\| \geqslant n+2$ and the pairs $T_{n}$ and $T_{n+1}$ satisfy $\left\|T_{n+1} T_{n} f\right\|^{2}=2\left\|T_{n} f\right\|^{2}-\|f\|^{2}$, for $f \in \mathcal{H}$ and $n \geqslant 0$. Hence, by Proposition 2.5, $T$ is the unbounded 2-isometry with an invariant domain. Notice that in the case in which the point spectra of $A_{00}$ and $V_{01}$ are empty sets, $T$ is not unitarily equivalent to any orthogonal sum of scalar weighted shifts (cf. Example 5.1).

AcKnowledgement. The author would like to thank Professor Jan Stochel for helpful discussions concerning the subject of the paper.

\section{REFERENCES}

1. A. Athavale, On completely hyperexpansive operators, Proc. Amer. Math. Soc. 124 (1996), 3745-3752.

2. S. K. Berberian, Notes on spectral theory (Van Nostrand, 1966).

3. N. Ivanovski, On a problem of a subnormal extension of operator valued weighted shifts, God. zbor. Matem. fak. 32 (1981), 37-40.

4. Z. J. Jabłoński and J. Stochel, Unbounded 2-hyperexpansive operators, Proc. Edinburgh Math. Soc. (2) 44 (2001), 613-629.

5. Z. J. Jabłoński, Complete hyperexpansivity, subnormality and inverted boundedness conditions, Integral Equations Operator Theory 44 (2002), 316-336.

6. R. Lee Kelly, Weighted shifts on Hilbert space, Doctoral thesis (University of Michigan, Ann Arbor, 1966).

7. A. Lambert, Unitary equivalence and reducibility of invertibly weighted shifts, Bull. Austral. Math. Soc. 5 (1971), 157-173.

8. W. Mlak, Dilations of Hilbert space operators (general theory), Dissertationes Math. 153 (1978), 1-61. $205-220$

9. S. Richter, Invariant subspaces of the Dirichlet shift, J. Reine Angew. Math. 386 (1988),

10. J. G. Stampfli, Which weighted shifts are subnormal? Pacific J. Math. 17 (1966), 377-379.

11. J. Stochel, Decomposition and disintegration of positive definite kernels on convex $\star$-semigroups, Ann. Polon. Math. 56 (1992), 243-294.

12. V. M. Sholapurkar and A. Athavale, Completely and alternatingly hyperexpansive operators, J. Operator Theory $\mathbf{4 3}$ (2000), 43-68. 